\title{
LAS AVANZADAS MINERO-AURIFERAS EN EL MACIZO COLOMBIANO: CONFLICTO, ASOCIATIVIDAD Y GOBERNANZA DEL TERRITORIO*
}

\author{
Recibido: 25 de diciembre del 2016 • Aprobado: 31 de octubre del 2018 \\ https://doi.org/10.22395/seec.v21n49a1
}

\author{
Raúl Cortés Landázury ${ }^{* *}$
}

\section{RESUMEN}

Este trabajo muestra las formas de articulación de los actores sociales tras las incursiones mineras y el legado de las movilizaciones de protesta de la última parte del siglo XX (1990-2000) en el municipio de La Vega (Cauca, Colombia). Para ello, se usó el análisis documental junto a una encuesta que funge como herramienta de Análisis de Redes Sociales (ARS); se tipificaron los indicadores y valores esperados y se construyó el grafo correspondiente para rastrear el conflicto y la gobernanza. Al final se encontraron nuevas formas de tramitar el fenómeno con fuerte arraigo en las organizaciones campesinas y arreglos socio-céntricos que, si bien generan conocimiento mutuo, requieren de conexiones multiescalares que superen arreglos endogámicos virtuales.

\section{PALABRAS CLAVES}

Extractivismo; movimiento social; recursos naturales; desarrollo.

\section{CLASIFICACIÓN JEL}

\author{
$\mathrm{O}_{1}, \mathrm{O}_{0}, \mathrm{Z}_{1}$
}

\section{CONTENIDO}

Introducción; 1. El contexto socioeconómico regional; 2. Conflicto ambiental vs gobernanza: perspectivas sobre el asunto; 3. Metodología; 4. Resultados; 5. Conclusiones; Bibliografía; Anexos.

- El presente documento recoge los resultados de la investigación realizados por el Grupo Desarrollo y Políticas Públicas Polinomía, clasificación Colciencias C, del proyecto denominado "De las dinámicas institucionales al desarrollo sostenible regional: la economía política del agua y la degradación en cuenca alta del río Cauca". Este se registró en la Vicerrectoría de investigaciones de la Universidad del Cauca bajo el código ID 3697 con fecha 21/04/ 2015 y culminación en febrero del 2016. El autor agradece el apoyo brindado por la Universidad del Cauca a través de su Vicerrectoría de Investigaciones y al economista Cristhian Guerrero por sus críticas, aportes y valiosas observaciones.

* Economista, Universidad del Valle, Santiago de Cali, Colombia. Especialista en Gestión Ambiental, Universidad Autónoma de Occidente, Santiago de Cali, Colombia. Magíster en Estudios Políticos Universidad Javeriana, Santiago de Cali, Colombia. Doctor en Economía de los Recursos Naturales y Desarrollo Sostenible, Universidad Autónoma de México, México. Profesor titular, Departamento de Ciencias Económicas, Facultad de Ciencias Contables, Económicas y Administrativas, Universidad del Cauca, Popayán, Colombia. Investigador, Grupo de Investigación Desarrollo y Políticas Públicas Polinomía. Carrera $2^{\circ}$ Calle 15n Esquina Campus Tulcán, Popayán, Cauca. Colombia. Correo electrónico: rcortes@unicauca.edu.co 


\title{
THE MOVE FORWARD OF GOLD MINING IN THE COLOMBIAN MASSIF: CONFLICT, ASSOCIATIVITY AND TERRITORY GOVERNANCE
}

\begin{abstract}
This paper presents the ways in which social actors articulate after the mining incursions and the legacy of protest mobilizations that occurred during the last part of the $20^{\text {th }}$ Century (1990-2000) in the municipality of La Vega (Cauca, Colombia). To do this, documentary analysis was applied with a survey that served as a tool for Social Network Analysis (SNA). Indicators and expected values were typified, and the corresponding graph was constructed to track conflict and governance. As a result, new ways were found to process the phenomenon which has strong roots in peasant organizations and socio-centric arrangements that, although generate knowledge, require multi-scalar connections that overcome virtual inbreeding arrangements.
\end{abstract}

\section{KEYWORDS}

Extractivism; social movement; natural resources; development.

\section{JEL CLASSIFICATION}

$\mathrm{O}, \mathrm{Q} 0, \mathrm{Z1}$

\section{CONTENTS}

Introduction; 1. Regional socioeconomic context; 2. Environmental conflict vs. governance: perspectives on the matter; 3. Methodology; 4. Results; 5 . Conclusions; Bibliography; Annexes.

\section{AS INCURSÕES MINERO-AURÍFERAS NO MACIÇO COLOMBIANO: CONFLITO, ASSOCIATIVIDADE E GOVERNANÇA DO TERRITÓRIO RESUMO}

Este trabalho mostra as formas de articulação dos atores sociais diante das incursões mineiras e do legado das mobilizações de protesto da última parte do século XX (1990-2000) no município de La Vega (Cauca, Colômbia). Para isso, a análise documental foi usada juntamente com uma enquete que funciona como ferramenta de Análise de Redes Sociais (ARS), os indicadores e os valores esperados foram tipificados e o diagrama correspondente para rastear o conflito e a governança foi construído. Ao final, foram encontradas novas formas de tramitar o fenômeno com forte arraigo nas organizações rurais e arranjos sociocêntricos que, apear de gerar conhecimento mútuo, requerem conexões multiescalares que superem arranjos endogâmicos virtuais.

\section{PALAVRAS-CHAVE}

Extrativismo; movimento social; recursos naturais; desenvolvimento.

\section{CLASSIFICAÇÃO JEL} $\mathrm{O} 1, \mathrm{Q} 0, \mathrm{Z} 1$

\section{CONTEÚDO}

Introdução; 1 . O contexto socioeconômico regional; 2. Conflito ambiental vs. governança: perspectivas sobre o assunto; 3. Metodologia; 4. Resultados; 5. Conclusões; Bibliografia; Anexos. 


\section{INTRODUCCIÓN}

América Latina ha transitado por un convulsionado periodo de conflictos sociales caracterizado por disputas en torno a las actividades de extracción y apropiación de los recursos naturales, pero el asunto nunca había comprometido tanto la sostenibilidad de los entornos locales como ahora. Con el advenimiento de la década de los noventa, el modelo de ajuste estructural puso las bases para que se acentuaran la dependencia externa y la sumisión a los marcos institucionales foráneos, los cuales en las siguientes dos décadas, hicieron proliferar las industrias extractivas como lo señalan Bottaro y Sola (2012) . En contraste, para Cordeiro (1995) también confluyeron la ampliación de mecanismos formales de participación que acompañaron andamiajes de descentralización política administrativa, la aparición de gobiernos de izquierda (progresistas), defensores de las causas sociales y promotores de grupos de base.

En Colombia, a los ya añejos conflictos políticos exacerbados por el narcotráfico y la violencia política, se han venido sumando los que comprometen la soberanía territorial, la disponibilidad de recursos naturales y la seguridad alimentaria. En este sentido, el macizo colombiano', fuera de ser el venero del agua que consume la mayoría de la población nacional, se erige en el teatro de los más fuertes conflictos que comprometen la zona que integra el municipio de La Vega en el departamento del Cauca. ${ }^{2}$ Por su ubicación estratégica (sureste del país), el modelo de desarrollo y el proyecto de modernización de principios de los noventa profundizó la inequidad y cercó a la pequeña propiedad rural, negando su ascenso productivo y de repartición de la riqueza, frente a lo cual los campesinos e indígenas de la zona se manifestaron en las postrimerías del siglo XX.

Más adelante, en la segunda década del siglo XXI, el problema se reeditó. Esta vez, con la presencia de explotación minera aurífera en la rivera de las fuentes hídricas vecinas del municipio. Bajo este renovado escenario y la herencia de las movilizaciones sociales de las décadas de 1980 y 1990, la pregunta es ćqué tipo de reacomodos asociativos y de gobernanza se han producido tras los conflictos ambientales y la defensa de la sostenibilidad del territorio en los últimos diez años comprendidos entre 2006 y 2016 ?

Llamado estrella fluvial (suroriente del país) o nudo de Almaguer.

2 Comprende 26 municipios de los departamentos de Nariño, Cauca y Huila, que conforman el núcleo del macizo colombiano y cuya extensión aproximada es de 1.452 .000 hectáreas que equivalen a casi el 50 por ciento de todo el departamento del Cauca, al 30 por ciento del de Huila y al 20 por ciento del de Nariño. Es más, la zona surte el agua del $70 \%$ de los acueductos del país, toda vez que el río Magdalena y el Cauca vierten sus aguas hacia el norte; el Caquetá viaja hacia el oriente y el río Patía que lleva el líquido hacia el occidente. 
Una conjetura que puede desprender sobre el particular es que la agudización de la violencia en la segunda década de 1990, con la intrusión de grupos guerrilleros -como las Fuerzas Armadas Revolucionarias de Colombia (FARC-EP) y el Ejército de Liberación Nacional (ELN)-, junto a la ofensiva de los grupos paramilitares y las fuerzas del Estado inspirados por la política de seguridad democrática pudieron haber opacado la acción colectiva ${ }^{3}$. Asimismo, el realineamiento de los partidos tradicionales, que atomizó el régimen político-electoral (transfuguismo político) en la primera década del 2000 (Congreso de la República, 2003; 2009), fragmentó la cohesión, lo cual virtualmente fatigó el accionar de la movilización social y las formas de gobernanza y desembocó en arreglos monocéntricos ${ }^{4}$. No obstante, el objetivo de este documento se centra en determinar las nuevas transacciones asociativas tras las mencionadas movilizaciones, dentro de los cambios en el modelo de desarrollo, y su grado de mutabilidad hacia esquemas multiescalares, policentrados o multinivel.

En este orden de ideas, el derrotero para rastrear el tema parte de revisar la historia de la movilización en las últimas dos décadas del siglo XX mediante análisis documental, para luego analizar la transición del conflicto ambiental hacia algún tipo de gobernanza multinivel con ayuda del Análisis de Redes Sociales (ARS). Las redes sociales (RS) y el ARS como representación y medida de las formas de articulación respectivamente, dan cuenta de las ligaduras comunicacionales que se forman

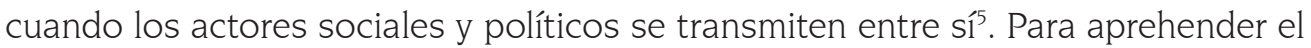
fenómeno, se utilizó una encuesta (ver anexo 1) que buscaba identificar vínculos entre organizaciones no gubernamentales (fundaciones, corporaciones) y demás grupos comunitarios, entidades privadas y públicas, que junto con la categorización y los valores esperados, sirvieron para hacer algunos cálculos (v. g., densidad, centralidad, intermediación) y gráficos sobre la naturaleza relacional de los agentes involucrados.

De esta manera, el engranaje de los actores, en caso de que funjan como puentes en la trama de la interacción, permiten examinar cómo transcurre la legitimidad del

3 Solo para hablar de incursiones guerrilleras, según datos del Centro de Memoria Histórica (2016) entre 1965 y el 2013, casi la mitad de los municipios con un mayor número de incursiones guerrilleras pertenece al departamento del Cauca. Especialmente en la zona Andina, en la cual destacan los casos en Toribio, Almaguer, Balboa y Bolívar.

4 En ambas reformas, tramitadas durante el gobierno de Á. Uribe, se prohibió la doble militancia de los ciudadanos, pero se dio la posibilidad a los miembros que hacían parte de las corporaciones públicas de cambiarse de partido, habiendo sido elegidos al inicio del periodo por un partido especifico, sin incurrir en ninguna sanción ni en la pérdida de la curul, hecho que vulneraba la elección de los ciudadanos y afectaba la representatividad de los partidos políticos en las corporaciones públicas.

5 Para estos propósitos, se aplicó una encuesta durante el segundo semestre del 2015 (noviembre 3, 2015) que inició con las 15 organizaciones más antiguas registradas en la Secretaría de Agricultura del Departamento del Cauca. A partir de estas, siguiendo el procedimiento de muestreo tipo "bola de nieve", se fueron relacionando paulatinamente e iteradamente, las nuevas entidades comparecientes que resultaran vinculadas con cada una de las inmediatamente anteriores. 
sistema de gobernanza, sus elementos procedimentales y la forma en la cual se asume el cambio a partir de los diferentes lenguajes de administración de los recursos de uso común, las expectativas compartidas y aún las divergentes y competitivas, tal como lo señalan Horning, Bauer y Cohen (2016).

A la postre, proliferaron nuevas formas de tramitar el conflicto que, si bien acumulan conocimiento endógeno, ostentan todavía baja densidad y diversidad de actores. Otros autores han podido registrar fenómenos análogos en la medida en que los países del sur son los más amplios y profundamente afectados, pero, aunque la producción intelectual sobre el particular ha redundado en zonas de Asia, África y América Latina, no hay mucha documentación sobre Colombia, y en particular, el macizo colombiano; se esquiva así, la complejidad de la zona y las implicaciones sobre la sostenibilidad del desarrollo nacional.

La mayoría de estudios ahondan en la utilización de diseños cualitativos valiosos, entre otras cosas en la exploración de las subjetividades. De esto dan cuenta las elaboraciones de autores como Avcl (2015) y Walter (2008). Esta investigación, sin embargo, agrega el tratamiento cuantitativo y relacional de los agentes para rastrear las formas asociativas de asumir el problema.

Este trabajo está divido en cinco partes. En la primera se describe el contexto de la problemática. Luego se exploran lineamientos teóricos en torno al conflicto y la gobernanza. De forma posterior, se expone la metodología, se señalan los indicadores principales del comportamiento de la red, tales como densidad y la centralidad con los signos esperados. Acto seguido, se explican los resultados y finalmente se concluye.

\section{EL CONTEXTO SOCIOECONÓMICO REGIONAL}

A principios de los noventa, el Estado colombiano y de manera subsidiaria, el departamento del Cauca, dio un nuevo aliento económico a zonas con virtual abundancia de recursos naturales, so pena de dar la espalda a las ventajas comparativas que ofrecían los mercados externos. Tres siglos antes, la comarca y la zona del macizo tenían protagonismo en el plano económico colonial por encontrarse en el camino real entre Popayán, Almaguer y Quito. Destacaba por ser un real de minas con enormes yacimientos de oro (Molano, 2011; Varela, 2014) ${ }^{6}$. A mediados del siglo XIX su base productiva fue la parcela campesina de mestizos, indígenas, y negros, por lo que empezó a aflorar un conflicto entre dos lógicas distintas: por un lado, la

La ciudad de Almaguer, fundada en 1550 y que en aquella época abarcaba municipios como La Sierra y La Vega, llegó a tener 2.000 indios y negros trabajando en ella. De manera que la región fue minera en la Conquista y en la Colonia, al decir de Molano (2011). 
tensión entre una economía minifundista y de subsistencia; frente a otra, latifundista de enclave, con ascendiente señorial y de alto estatus simbólico colonial. En este orden de ideas, habría bajas aspiraciones de inserción internacional, pero altos réditos político-electorales.

El siglo XX no sería ajeno a esta tendencia, con un ingrediente adicional: políticas estatales zambullidas en un modelo de desarrollo que establece subsidios focalizados en detrimento de los pequeños productores y de las comunidades rurales. Ya entrado el siglo XXI, a esta trama se le sumó la que opondría a la dinámica parcelaria y de pequeña empresa, y la de un gigantesco proyecto de explotación minera de origen externo que evoluciona a la vera de pequeños polos de explotación. La irrupción tiene convulsionada la zona, una región agreste, de difícil topografía que contrasta con la gran oferta natural de su paisaje ${ }^{7}$.

El municipio de La Vega, (departamento del Cauca) es el municipio más extenso de esta vasta subregión y no ha sido ajena a las realidades de los otros municipios del macizo. En particular, los actores de la confrontación forman un núcleo que vincula a los moradores, los inversionistas y el Estado y, en la periferia, a cazadores de rentas y grupos al margen de la ley. En un primer momento, es importante resaltar el auge que ha tenido la minería ilegal de tipo socavón, jalonada por las trepadas de precios internacionales; y en un segundo momento, las licitaciones de las multinacionales para la exploración y explotación de oro en minas a cielo abierto, tal como lo señalan Duarte et al. (2013) y Duarte et al. (2014, p. 317)

No obstante, desde el punto de vista de la planeación del desarrollo, los proyectos minero-auríferos, enmarcados en lo que se denominó las locomotoras del desarrollo, pretendieron privilegiar la rentabilidad privada, seguida de la apropiación tributaria del Estado con facultad del gobierno central para la posterior redistribución vía regalías (Cortés y Gómez, 2015; Botero et al. 2015). Bajo este escenario, se desarrolla una lógica de apropiación medular tipo enclave en la cual el gobierno central reparte las ganancias a las zonas marginadas, aunque no sean generadoras directas de la riqueza, en lo que comporta el modelo de descentralización político administrativo. A la postre, los impactos ambientales negativos sobre los sistemas

Se trata además del principal nudo geográfico de la cordillera de los Andes en Colombia, en la que se originan las vertientes de las cordilleras central y oriental, por lo que se puede caracterizar como una ecorregión. Este, a su vez, se constituye en el punto de confluencia de 5 de las 6 eco-regiones estratégicas, definidas por el Banco Mundial y el World Wildlife Fund (WWF).

8 Por ello la multinacional de origen sudafricano Anglogold Ashanti, junto con la canadiense B2Gold, en febrero del 2008 anunciaron que extraerían oro, cobre y molibdeno en dos municipios: La Vega (Cauca) y Mocoa (Putumayo). No obstante, en el departamento del Cauca existen 350.447 ha en título de mineros, según la Agencia Nacional de Minería (ANM) (2012), que hacen parte de las 369.111,83 ha destinadas para la explotación de recursos naturales no renovables totales para el departamento. 
socio-ecológicos se intentan paliar con un plan de manejo ambiental o con la instrucción de una tasa impositiva por parte del Estado a los inversionistas.

Vale anotar que en la constitución colombiana el subsuelo no pertenece a los lugareños y se puede concesionar a un privado. Para los territorios en donde se asientan comunidades étnicas, la cuestión no procede de forma directa, porque las explotaciones no consideran el mecanismo de consulta previa9. No obstante, para casos como este, siendo la población en su mayoría mestiza, las zonas de explotación no están sujetas a la norma contenida en este mecanismo; razón por la cual la sostenibilidad y gobernanza del territorio se desenvuelve en forma distinta (Duarte et al., 2013).

Según analistas como Calderón (2012) y Castro et al. (2015), América Latina ha propiciado una corriente de movilización originada en conflictos ambientales producto de la extracción de recursos, expansión de vertederos y riesgos de contaminación para quienes el ambiente es la base material de sustento. En este sentido, se puede pensar también que las movilizaciones sociales de finales de las décadas de 1980 y 1990 respondieron a esta suerte de alteraciones, como quiera que reclamaban soberanía alimentaria, equidad y autocontrol de los recursos naturales. De esto da cuenta, según Mamián et al. (2013), la primera movilización efectuada en 1987, liderada por municipios como Santa Rosa y Bolívar y que luego, escaló con el bloqueo de la carretera Panamericana en 1999. Todas estas se muestran coincidentes con voces contestarías de países del sur como México, Argentina y Perú10 ${ }^{10}$

En efecto, en el ámbito regional el movimiento logró movilizar más de 10.000 marchantes que condujeron al final a una negociación, que, si bien para algunos no fue significativa en términos de consecución de recursos, sí lo fue desde el punto de vista político. Después de un bloqueo de más de veinte días en la carretera Panamericana, el movimiento dejó su impronta en las arenas del poder, según lo narra Tocancipá (2005). Por ello, desde que el Movimiento del Macizo Colombiano (MMC) puso en escena la importancia de esta zona de vida como un territorio estratégico para la conservación de recursos naturales, el país pudo identificar el grado

9 De acuerdo a la Sentencia SU-039 de 1997 de la Corte Constitucional (1997), la consulta previa es el derecho fundamental que tienen los pueblos indígenas y los demás grupos étnicos cuando se toman medidas (legislativas y administrativas) o cuando se vayan a realizar proyectos, obras o actividades dentro de sus territorios, busca de esta manera proteger su integridad cultural, social y económica y garantizar el derecho a la participación.

10 No sobra advertir que el término países del sur, corresponde a todos aquellos países que por circunstancias históricas no hicieron parte del club de las naciones desarrolladas, por extensión del quiebre que se produjó en 1954 tras la Guerra Fría. De esta forma, el mundo se dividió entre potencias con grandes despliegues económicos y financieros y aquellos que no calzaban en ese grupo (países no alineados). 
de afectación que revestiría el desconocimiento de la dinámica socio-ecológica, aunque no se eliminaran sospechas de su conexión con las estrategias guerrilleras, de acuerdo con lo planteado por Duarte et al. (2013).

Entre 1996 y el 2000, casi que se incrementaron el número de manifestaciones, alcanzaron su punto máximo en el 2000, para luego declinar al comienzo del nuevo siglo. De allí en adelante, empezó un periodo de aletargamiento, explicado por la dispersión y las disidencias, pero también por los nuevos arreglos institucionales que vivía el país en materia política y de orden público. Sin embargo, los fenómenos globales y regionales siguieron adelante. La cuestión entonces que se ha de discutir es si el prototipo de escenario conflictual y los niveles de transformación en materia de movilización, pudieron generar arreglos asociativos nuevos y complementarios frente a herramientas como los taponamientos viales. De allí, la necesidad de explorar el tema bajo categorías distintas de relacionamiento.

\section{CONFLICTO AMBIENTAL VS GOBERNANZA: PERSPECTIVAS SOBRE EL ASUNTO}

Desde la teoría económica imperante, el punto de partida para el análisis de fenómenos como el que comporta la explotación minera en el macizo colombiano se resolvería en términos de externalidades. Por ejemplo, en el enfoque neoclásico se explican las externalidades como efectos negativos (o a veces positivos) no recogidos en los precios del mercado ${ }^{11}$. Mientras tanto, algunas tesis alimentan la idea de que el crecimiento de las economías resulta en una mejora de las condiciones ambientales (Baumol y Oates, 1971). En contraste, otras le atribuyen el origen de estos a fenómenos ligados a los procesos conflictivos que rondan las asimetrías redistributivas y de poder (Hogenboom et al., 2014). Pero más allá de considerarlos averías al régimen de propiedad privada por la no incorporación en los costos que soportan los precios; la dinámica de estas problemáticas no solo convoca factores exógenos (como los activistas de organizaciones ambientalistas) al Estado y a las empresas, sino a los ecosistemas y comunidades afectadas por un proyecto de extracción de recursos naturales que en su entorno, recrea la nueva fase de fijación de la acumulación capitalista en los países del sur, al parecer de Martínez-Alier (2006)

Si el asunto rebasa la esfera de la movilización o los movimientos sociales, hace pensar en estos como sucesos enquistados a su vez en la hechura de las políticas del desarrollo, cuestión que deriva en problemas de gobernanza, un fenómeno que

\footnotetext{
11 Otros autores como Stiglitz, (2010) y Skorková, (2016) también ven las externalidades como "fallos del gobierno", o fracasos del sector público, que no se ponen de acuerdo para establecer normas ambientales internacionales, o que no aciertan a imponer una estructura de derechos de propiedad sobre el ambiente, o que subsidian actividades nocivas. Así, lo agregan Stiglitz, (2000) y Callan (2007)
} 
registran autores como Merino (2015) y Correa y Rodríguez (2005). Para autores como Walter (2006), si bien por décadas se ha hablado de los problemas ambientales como equivalentes a los conflictos ambientales, su origen gira alrededor de la colisión de intereses, una cuestión que contrasta con el nivel de identificación neoclásica. Para ello, solo basta pensar en las características biofísicas, los rasgos de los actores e incluso hasta la temporalidad de los fenómenos. Es más, el mismo término conflicto, difiere del de problema ambiental, dado que, desde la perspectiva etimológica, la palabra conflicto proviene del latín conflictus que significa choque.

Por lo tanto, confluye en una situación en la que un(os) actor(es) se encuentran en oposición consiente a otro(s) actor(es), que pueden ser persona(s), grupo(s), organizaciones sociales o instituciones. Al respecto, según Martínez-Alier (2004), los actores comportan disputas ecológico-distributivas y esto los hace agentes inmersos en el tratamiento de la ecología política. De esta forma, el conflicto ambiental supone una diferencia posicional con respecto a algún valor o conjunto de valores relevantes para una sociedad. Por su parte, siguiendo a Ille (2016), también involucra al menos dos actores que se debaten frente a la posesión o no de algún objeto, los directamente implicados llevan a cabo medidas observables e interrelacionadas, que se encuentran por fuera de los procedimientos de regulación y que ponen en peligro las funciones básicas del Estado, el orden internacional o su decisión de debatirlo.

Sin embargo, los conflictos socioambientales son conflictos sociales relacionados con las actividades extractivas que no necesariamente derivan de problemas del diseño de las políticas, sino de divergencias que trascienden el ejercicio temporal del gobierno y se expresan a manera de diferentes ontologías políticas. Es decir que, particularmente encarnan una interpretación sobre los vínculos entre el ambiente y la sociedad que refleja determinados valores y relaciones de poder, posición que comparten Martínez-Alier (2006) y Mondéjar (2015).

Ahora bien, según el tipo de intereses a debatir, el abanico de posibilidades -de acuerdo con su origen (mediación o metabolismo social)-, pueden derivar de problemas de información, de la naturaleza de las relaciones, o de intereses, estructuras y valores, en el caso de los mecanismos de mediación u objeto de divergencia. De acuerdo con la etapa del proceso productivo involucrado, puede ser de extracción, transporte o vertimiento de residuos. Para el caso que nos ocupa, los tipos de conflictos involucrados parecen encajar en los de mediación y dentro de estos, conflictos por relaciones, en la medida en que contemplan la desconfianza generada por incumplimientos del Estado que redundan en las movilizaciones (ver tabla 1). 
Tabla 1. Clasificación de los conflictos ambientales

\begin{tabular}{|c|c|c|c|}
\hline Tipo de conflicto (a) & Origen & & Tipo de conflicto (b) \\
\hline & Mediación & & $\begin{array}{l}\text { etabolismo social } \\
\text { ommodity chain) }\end{array}$ \\
\hline Por información & $\begin{array}{l}\text { Carencia de información o } \\
\text { el uso de técnicas de reco- } \\
\text { lección. }\end{array}$ & Extracción & $\begin{array}{l}\text { Sobre extracción de mate- } \\
\text { riales y energía. (ej.: minería } \\
\text { metalífera, petrolera, de } \\
\text { cantera, etc.) }\end{array}$ \\
\hline Por relaciones & $\begin{array}{l}\text { Desconfianza, } \\
\text { la falta de credibilidad o la } \\
\text { duda sobre la integridad de } \\
\text { las partes }\end{array}$ & $\begin{array}{l}\text { Transporte y } \\
\text { tratamiento de } \\
\text { los residuos }\end{array}$ & $\begin{array}{l}\text { Movimiento de materiales y } \\
\text { energía y sus impactos. } \\
\text { (ej: derrames petroleros, } \\
\text { accidentes en oleoductos o } \\
\text { gasoductos) }\end{array}$ \\
\hline Por intereses & Disputas sobre posiciones & \multirow{3}{*}{ Residuos } & \multirow{3}{*}{$\begin{array}{l}\text { Vertimiento, generación y } \\
\text { tratamiento de efluentes (ej. } \\
\text { asbestos, dicloro difenil tri- } \\
\text { cloroetano (DDT), organis- } \\
\text { mo genéticamente modifi- } \\
\text { cado (OGMs)). }\end{array}$} \\
\hline Estructurales & $\begin{array}{l}\text { Cuando hay límites físicos, } \\
\text { institucionales o formales }\end{array}$ & & \\
\hline Por valores & $\begin{array}{l}\text { Distintos sistemas de creen- } \\
\text { cias }\end{array}$ & & \\
\hline
\end{tabular}

Fuente: síntesis de elaboración propia con base en Walter (2006) y Martínez-Alier (2006)

Asimismo, abarca los conflictos por valores, en tanto la dinámica del aprovechamiento del pancoger choca con la racionalidad económica maximizadora arrastrada por los programas del Estado y las incursiones de minería ilegal y/o foránea. Por el lado del metabolismo, claramente la tensión se presenta en la etapa de extracción que involucra la explotación del mineral, sin introducción de valor agregado y sin retornos financieros o de inversión a la zona de aprovechamiento, en lo que la literatura económica reconoce como economías de enclave, cuestión en la que coinciden Falero (2015), Barbier (2016) y Côte y Korf (2016) ${ }^{12}$.

Ahora, si se intentan definir nuevas formas de articulación y manejo del conflicto en términos de cohesión y acción colectiva, se puede concebir con Jessop (2008) la gobernanza como una trama compleja de interacción cuya finalidad es recoger la armonización económica, política y social en pos de mejores niveles de bienestar ${ }^{13}$. No obstante, en una dimensión más concreta sobre el asunto, permite

12 Hay que señalar que a largo plazo el auge de los precios de bienes primarios como el oro, que presionan la explotación en la región, obedece en parte al aumento de la demanda de China, lo cual habría originado un incremento de los precios de entre el $12 \%$ y el 52\% durante el periodo comprendido entre 1995 y el 2004.

13 Más adelante, Jessop (2008) amplía la cuestión al proceso que contempla el protagonismo del Estado, de la administración de las formas de autoorganización que permiten compensar los fallos, tanto de la planificación como del mercado, en una sociedad cada vez más conectada en redes. 
comprenderlo como un proceso por el cual un conjunto de organizaciones aborda y resuelve asuntos comunes bajo un acuerdo institucional a la usanza de Young et al. (2008). ¿Cómo entonces examinar el despliegue de este fenómeno en el macizo?

Uno de los factores identificados como importantes para la operación aceitada de los sistemas de gobernanza, de los cuales depende una gestión efectiva de recursos naturales, son los vínculos comunicacionales que se forman cuando los actores sociales y políticos se transmiten mensajes y se reúnen entre sí. La disposición dinámica de cualquier campo de acción social está marcada por diferentes capacidades de poder e intereses articulados a través de procesos históricos de estructuración de redes, tal como lo asumen autores como Ribeiro (2007). En consecuencia, su forma de articulación, más allá del acto coactivo, habla de las gamas de gestión del conflicto y el riesgo asociado a su desenvolvimiento, cosa que recoge Berardo (2016).

Una red social corresponde a un conjunto bien definido de actores, individuos, grupos, organizaciones, comunidades y sociedades globales que están vinculados unos a otros a través de una o un conjunto de relaciones sociales, dentro de lo planteado por Scholz et al. (2008) y Bodin y Crona (2009). La cuestión ahora es cómo plasmar el asunto en términos del plexo de relaciones virtualmente construidas para atender el conflicto.

\section{METODOLOGÍA}

Para Hanneman (1998), Brenner, (2010) y Alfie, (2013) las estructuras sociales pueden desplegar altos o bajos niveles de engranaje y gobernanza frente a escenarios conflictivos. Los resultados son variables dependiendo de los patrones que se tejan en materia de vínculos entre los actores. Siendo así, las redes sociales y el Análisis de Redes Sociales (ARS) recrean el espacio institucional en el cual distintos agentes se reúnen y generan interacciones delimitadas. De allí que la teoría de grafos, que asume su representación visual, constituye la base de las medidas de interacción en términos de ensanchamientos o rupturas de cooperación. El primer indicador atinente a esta clase de fenómenos es la densidad, que mide el número de vínculos existentes ${ }^{14}$, sobre todos los posibles en la red. Para este caso, los valores extremos son cero, para vínculos inexistentes y uno para articulaciones plenas (ver tabla 2).

En el sucesivo, se consideraron otras medidas como el grado de centralidad, la cercanía y la intermediación que evidencian el protagonismo de los intervinientes en el sociograma. Como se puede inferir de la tabla 2, una red es más monocéntrica o egocéntrica entre menos densa sea, mayor nivel de centralidad tenga y más

14 Vínculos son relaciones existentes entre los actores de la red (Hanneman, 2005). 
cercana se muestre. Si por el contrario llega a ser más prolífica, es decir más densa, menos centrada en un actor y que ostente menos intermediación, más policéntrica, multinivel y multiescalar será. Para los fines pertinentes, en este estudio se aplicó una encuesta (ver anexo 1) que indagó sobre los niveles de relacionamiento, a partir del registro de organizaciones sociales campesinas inscritas en la Secretaría de Agricultura del Departamento del Cauca para el 2015.

Tabla 2. Análisis de Redes Sociales: valores y signos esperados

\begin{tabular}{|c|c|c|c|c|}
\hline \multirow{2}{*}{ Indicadores } & \multicolumn{2}{|c|}{ Componentes } & \multirow{2}{*}{ Definición } & \multirow{2}{*}{$\begin{array}{l}\text { Comportamiento } \\
\text { esperado }\end{array}$} \\
\hline & Posición & Estructura & & \\
\hline $\begin{array}{l}\text { Densidad } \\
\text { (cohesión) }\end{array}$ & & $\mathrm{X}$ & $\begin{array}{l}\text { Número de vínculos que se establecen } \\
\text { entre los nodos con relación a un número } \\
\text { máximo que se pudiera establecer. }\end{array}$ & $0,5 \leq$ den $\leq 1$ \\
\hline $\begin{array}{l}\text { Grado de } \\
\text { centralidad } \\
\text { (o grado) }\end{array}$ & $\mathrm{X}$ & & $\begin{array}{l}\text { Número de conexiones directas (distancia } \\
\text { igual a 1) que tiene un actor con los demás. }\end{array}$ & $0 \leq \mathrm{Gc} \leq 0,5$ \\
\hline
\end{tabular}

Suma de las distancias que separan a un

actor del resto de actores de la red (indica

la capacidad de un actor para llegar a otros $\quad \mathrm{CD} \approx 0$

en pocos pasos).

Interme-

diación
La proporción de veces que un actor está

en la ruta entre distintos pares de actores.

$0 \leq \mathrm{I} \leq 0,5$

Fuente: elaboración propia con base en Bodin y Crona (2009) y Encuesta sobre conflictividad, redes sociales y gobernanza en La Vega (nov. 3, 2015)

Como la pregunta reina giraba alrededor de los lazos de colaboración frente a la defensa del agua y el territorio en forma de proyectos, mingas y convenios, tras las avanzadas mineras de finales de los noventa, el método de muestreo respondió al de bola de nieve. Consistió en revisar el tipo de articulación entre estas, siguiendo la cadena de alusiones de una entidad a otra a las que remitían iterativamente los miembros de los cuerpos directivos de las asociaciones, corporaciones, fundaciones y Organizaciones No Gubernamentales (ONG) entrevistadas. Cabe agregar que la trama de entrevistas que enlazaban terminó cuando los vínculos se mostraron redundantes.

Los datos se procesaron con el programa Ucinet (61.2) y se inició con entrevistas a ONG representativas de la región de aquella gran época de movilizaciones, como la Fundación de Integración del Macizo Colombiano (Fundecima) y el Proceso de Unidad Popular del Suroccidente Colombiano (Pupsoc). Con esto, se realizaron los 
gráficos correspondientes (grafos) $)^{15}$ que concernirían a virtuales arreglos cohesivos monocéntricos, con decisiones cifradas en un actor central; sociocéntricos, cuando se concentraron en un grupo de actores, pero en los que la información puede redundar; y policéntricos, con altos niveles de diversidad y conexiones externas a la zona o ámbito político de origen.

Con esta nueva información y la de las movilizaciones, se esperaba que la conflictividad socio-ecológica de la década de 1980 y sobre todo la de 1990, se hubiera alejado de los horizontes monocéntricos y que hubiera migrado en las casi primeras dos décadas del 2000, en arreglos policéntricos, con alta densidad y bajo nivel de protagonismos corporativos; con lo cual se habrían ampliado los procesos de autoactividad y acción colectiva; se habrían ensanchado las formas de gestión del conflicto ambiental y por supuesto, se habría alcanzado mayor gobernanza territorial.

\section{RESULTADOS}

El análisis pudo mostrar cuestiones paradójicas de la actividad asociativa frente a la amenaza del extractivismo y la minería a gran escala. En efecto, la densidad resultó baja, como quiera que las conexiones se extienden tan solo en 7,6\% dentro del 100\% de articulaciones posibles. Mientras que, dentro de la centralidad, el actor más visible, poderoso y prestigioso es la organización Somos Agua de esta Tierra, con un eingenvector de 57,13 que define el patrón principal de distancias entre todos los actores ${ }^{16}$. Un eingenvector mide la capacidad de influir de un nodo en la red, siendo más importante entre mayor sea su guarismo, cosa que un alto valor, indica que los actores son más centrales al patrón principal de distancias entre todos los demás. De esta forma, luego de Somos Agua de esta Tierra, siguen Wairasitay y Abriendo Trocha con un eigenvector del 56,46. No obstante, el indicador global de centralidad para la red es de $47,15 \%$, lo cual significa que, en total, las ventajas posicionales se distribuyen de forma desigual con un alto grado de centralidad en varias organizaciones sociales.

De otro lado, el indicador de cercanía que mide la distancia media de cada actor con respecto al resto ${ }^{17}$, pero discriminado por sus capacidades de recibir y transmitir

15 Los grafos son representaciones de las redes sociales, que relacionan nodos o actores en forma de puntos y vínculos en forma de líneas. Estos pueden ser dirigidos, según la relación unidireccional de un actor a otro; o no dirigidos cuando tienen doble sentido. En este estudio se elaboraron los esquemas respectivos con el programa Netdrw (5.0).

16 En el lenguaje matemático, son los vectores no nulos que, cuando los transforma el operador, dan lugar a un múltiplo escalar de sí mismos. En el ámbito de los análisis de redes sociales, si un nodo está unido a nodos muy importantes tendrá un eigenvector o relevancia más alta.

17 Un mayor valor en este indicador evidencia una gran capacidad para enviar y recibir información. 
información, mostró dos tipos de organización con altos niveles de proximidad. Por el lado de la capacidad de recibir información (incloseness) está el Proceso Campesino y Popular de la Vega (PCPV) con 6.667 intermediaciones, seguido del Proceso Unidad Popular del Suroccidente Colombiano (Pupsoc) con 6.250 y el Comité de Integración del Macizo Colombiano (CIMA) con 6.231. De otro lado, en cuanto a la capacidad de transmitir información a otra (outcloseness) o el poder de expandirse en otros vínculos, están las organizaciones Abriendo Trocha y Wairasitay con un 10.811, seguido de Somos Agua de esta Tierra con un 10.695 (Ver anexo 2).

Por su parte, el indicador de intermediación que revela la medida en que se encuentra cada actor frente a la interfaz de posición intermedia muestra a la organización social Conciencia Campesina como el actor de mayor intermediación con 9.000 vínculos. La siguen la organización Abriendo Trocha con 4.000 y con menores valores a Apimacizo, Somos Agua de esta Tierra y Wairasitay ${ }^{18}$. Además, los resultados arrojan a actores con un grado de intermediación igual a cero, lo que significa que no hay dependencia con los demás actores (v.gr. Asoprocomqui, Brisas del Macizo).

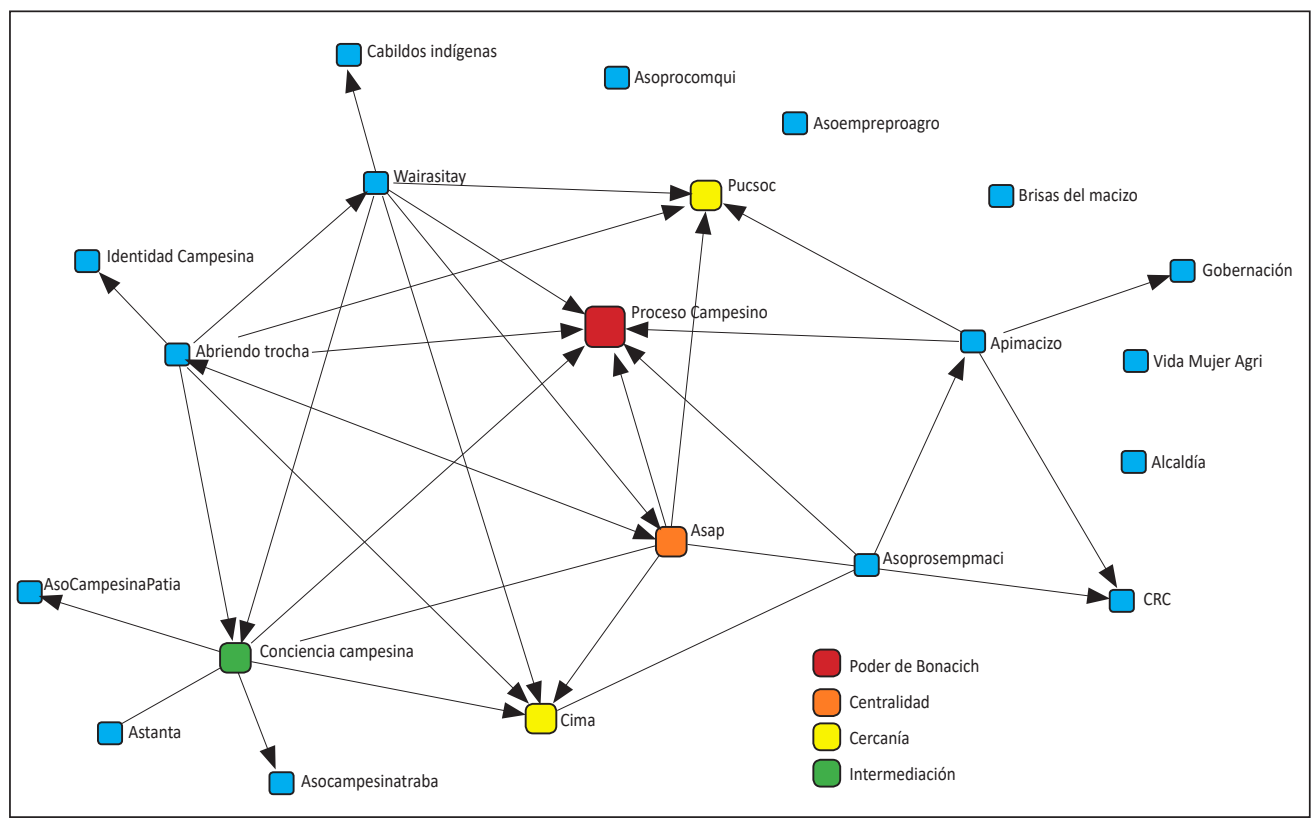

Figura 1. Grafo de organizaciones sociales y actores sociales - La Vega, Cauca

Fuente: elaboración de los autores a partir de encuesta aplicada y procesada en el programa UCINET.6 y la interfaz Netdraw 1.48

18 La distancia geodésica se refiere al número de nodos de distancia entre un actor y otro. 
En suma, tomando como punto de partida el municipio de La Vega (ver figura 1), el tipo de relacionamiento más pronunciado en la región es de tipo sociocéntrico, tal como lo tipifica Rodríguez (2013). Este se caracteriza por no estar estructurado en torno a un foco preciso, como lo hace la red egocéntrica, con un sujeto social difuso, pero que tiende a concentrar la acción colectiva en ramificaciones que no tienden puentes lejanos con otras organizaciones, instituciones, grupos y actores de afuera; que además puede concentrar la información y el poder en un puñado. Desde luego, destierra el prototipo de estrella monocéntrico (egocéntrico) poco deliberativo y cuasiautocrático (ver figura 1), pero no llega a ser tan amplio y flexible como el policéntrico. En efecto, al interior se distinguen como actores poderosos, el PCPV, seguido de cerca por el CIMA, y Pupsoc. El resto de organizaciones o actores carece de poder u ocupan un rol marginal.

Llama la atención, el aislamiento de entes de naturaleza estatal como la Alcaldía, la Secretaría del Medio Ambiente Municipal y la Corporación Autónoma Regional del Cauca (CRC) que estarían llamadas a intermediar en la problemática. De otra parte, sorprende el casi total aislamiento de los cabildos indígenas de la zona, donde la comunidad yanacona ${ }^{19}$ tiene alta presencia y esgrime como elemento de defensa territorial, la soberanía sobre el patrimonio ancestral allende a los recursos naturales. De igual manera, la total invisibilidad de organismos de cooperación internacional y ONG multilaterales que evidenciarían la articulación del tema como parte de un conflicto sistémico y mundial.

Así las cosas, los niveles de gobernanza en materia reticular y policéntrica parece un poco débil, aunque ostenta niveles de articulación entre unos cuantos actores que lideran los procesos y que podrían agilizar la toma de decisiones en el corto plazo, pero cuya principal amenaza es la redundancia de la información y con ello, la disposición de mayores recursos para sostener en el largo plazo la posición de defensa ante los grandes andamiajes de las corporaciones multinacionales y los actores armados presentes en la zona.

\section{CONCLUSIONES}

Si bien la dinámica del extractivismo promete transmitir expectativas de un mayor crecimiento económico presumiblemente local, sus extrapolaciones no han transformado el bienestar social de la población. Más bien, parecen aislar al macizo

19 El Pueblo Yanakuna o comunidad Yanacona, es un grupo indígena que habita en seis departamentos de Colombia, en su mayoría en el departamento del Cauca, principalmente en la zona del macizo colombiano. Los yanaconas se resistieron a la extinción cultural y física desde el siglo XVIII y XIX, y en la actualidad experimentan un proceso de reetnización, de recuperación de su identidad y de reconocimiento y reivindicación cultural, social, económica y política. 
colombiano de la gobernabilidad económica y socioambiental del resto del territorio nacional, se segrega así a la región en una especie de zona apropiada para el buen salvaje, que según retoma Yagüe (2010), además debe contribuir con el agua y sus recursos naturales para hacer méritos y lograr su inclusión en las agendas del desarrollo nacional ${ }^{20}$. Tampoco logra los réditos necesarios para vislumbrar cambios radicales en materia de gobernanza y sostenibilidad de la zona, dada la estrechez de los puentes externos y vínculos con las organizaciones inscritas en la región. A eso podrían estar contribuyendo la accidentalidad topográfica, el bajo nivel de control estatal y la presencia de grupos armados.

De contera, el desenvolvimiento de los escenarios de conflictividad y gestión de acciones colectivas tienden a la generación de una suerte de acumulación por desposesión (Harvey, 2005) asistida por el Estado colombiano a partir de legislaciones favorables y flexibles a la desterritorialización de los actores asentados en la zona. Empero, luego del empuje de las movilizaciones de las décadas de $1980 \mathrm{y}$ 1990, la configuración de redes de gobernanza, que han sido de corte sociocéntrico, congregó su fortaleza en el conocimiento de los actores dentro de una suerte de burbuja que permite ubicar la información en actores relevantes. No obstante, no ampliar y diversificar dicha burbuja puede conllevar un tipo de anquilosamiento que ralentice la acción colectiva por efecto de la redundancia de la información, que afloren los liderazgos mesiánicos y las capturas de rentas y pulule la desconfianza.

Los resultados no son radicalmente distintos a los hallazgos de otros autores como Avcı (2015), que hace un estudio comparativo para algunas zonas del Ecuador y Turquía y rastrea la dinámica del conflicto extractivo y la construcción de subjetividades alrededor del papel del Estado y las relaciones de resistencia entre los actores. Por su parte, Walter (2008) -para el caso de la Esquel, Argentina- y Sovacool y Andrews (2015) en Azerbaiyán, reconstruyen las características de la organización de una red de comunidades afectadas con base en el ciclo del conflicto: su inicio, sus puntos de inflexión, los actores, sus percepciones y lenguajes de valoración, para así considerar qué estuvo en juego en las diferentes etapas.

En general, los resultados asociativos aparecen endebles, en tanto se presentan una serie de disputas en torno a la información y la percepción del riesgo que socavan la confianza de la comunidad en las acciones colectivas y sus impulsores. Bajo este panorama, los resultados en el caso del municipio de La Vega parecen asintomáticos en esta etapa del desarrollo: se muestra baja capacidad de generar

20 El término, popularizado por J.J. Rousseau, se refería a la idealización de los pueblos primitivos y la apología de la vida salvaje. En efecto, el hombre natural sería originariamente íntegro, biológicamente sano y moralmente recto; por lo tanto, no malvado, ni opresor e injusto. 
vínculos tipo puente, dificultades en la articulación de múltiples actores y un tinte de desconfianza que, si bien comanda las acciones colectivas hoy, amenaza la cohesión interna hacia el futuro. No obstante, la madurez del proceso podría potencializar las acciones colectivas hacia una mejor administración del conflicto que propicie mejores escenarios de negociación en el ambiente de aperturas y reacomodos que permite la globalización.

\section{BIBLIOGRAFÍA}

Agencia Nacional de Minería (2012). Relación de títulos otorgados en el año 2012, 236p.

Alfie Cohen, Miriam. (2013). Democracia deliberativa y gobernanza ambiental: ¿conceptos transversales de una nueva democracia ecológica? En: Sociológica (México), Vol. 28, No. 80, p. 73-122.

Avc1, Duygu (2015). Mining conflicts and transformative politics: A comparison of Intag (Ecuador) and Mount Ida (Turkey) environmental struggles. En: Geoforum, Vol. 84, p. 316-325. doi: https://doi.org/10.1016/j.geoforum.2015.07.013

Barbier, Edward B. (2016). Is green growth relevant for poor economies? En: Resource and Energy Economics, Vol. 45, p. 178-191.

Baumol, William y Oates, Wallace (1971). The use of standards and prices for protection of the environment. En: Swedish Journal of Economics. Vol. 73, No. 1, p. 42-54.

Berardo, Ramiro (2016). Redes y percepción de riesgo en sistemas socioecológicos. En: Edgar Ramírez (ed.), Análisis de redes sociales para el estudio de la gobernanza y las políticas públicas. México D.F: Centro de Investigación y Docencia Económicas, CIDE, p. 157-190.

Bodin, Örjan y. Crona, Beatrice (2009). The role of social networks in natural resource governance: What relational patterns make a difference? En: Global Environmental Change. Vol. 19. No. 3. p. 366-374. https://doi.org/10.1016/j.gloenvcha.2009.05.002

Botero, María Elena; Hofman, Juana Marina; Hernández, Daniel Eduardo y Pico, María Juliana (2015). Regalías y desequilibrios territoriales en Colombia: una brecha que no se cierra. Documento de investigación No. 47. Universidad del Rosario, Bogotá: CEPI, 58p.

Bottaro, Lorena y Sola Álvarez, Marian (2012). Conflictividad socioambiental en América Latina: El escenario post crisis de 2001 en Argentina. En: Política y cultura, No. 37, enero, p. 159-184.

Brenner, Ludger (2010). Gobernanza ambiental, actores sociales y conflictos en las Áreas Naturales Protegidas mexicanas. En: Revista mexicana de sociología, Vol. 72, No. 2, p. 283-310.

Calderón, Fernando (2012). Diez tesis sobre el conflicto social en América Latina. En: Revista Cepal, No. 107, p. 7-30.

Callan, Scott., y Thomas, Janet (2012). Environmental Economics and Management: Theory, Policy, and Applications. Boston, United States of America: Cengage Learning, 626p.

Castro Fabio de, Hogenboom, Barbara y Baud, Michiel (coords.) (2015). Gobernanza ambiental en América Latina. Ciudad Autónoma de Buenos Aires: CLACSO; ENGOV, p. 376. 
Cataño, José Felix (2004). La teoría neoclásica del equilibrio general. Apuntes críticos. En: Cuadernos de Economía, Vol.23, No 40, enero-junio, p. 175-204.

Centro Nacional de Memoria Histórica. (2016). Tomas y ataques guerrilleros (1965-2013). Informe del Centro Nacional de Memoria Histórica (Martha J. Espejo Barrios). Bogotá, Colombia: Universidad Nacional de Colombia, 495 p.

Congreso de la República (2003). Acto legislativo 01 de 2003. Por el cual se adopta una Reforma Política Constitucional y se dictan otras disposiciones, 13p.

Congreso de la República (2009). Acto legislativo 01 de 2009. Por el cual se modifican y adicionan unos artículos de la Constitución Política de Colombia, 6p.

Cordeiro, José Luis (1995). El Desafío Latinoamericano y sus cinco grandes retos. Caracas, Venezuela: McGraw-Hill, 319 p.

Correa, Francisco (2003). Economía del desarrollo sostenible: propuestas y limitaciones de la teoría neoclásica. En: Semestre Económico, Vol.6, No 12, p. 1-21.

Correa, Hernán Darío y Rodríguez, Iokiñe (2005). Encrucijadas ambientales en América Latina. Entre el manejo y la transformación de conflictos por recursos naturales. Costa Rica: Universidad para la Paz, 395 p.

Corte Constitucional de Colombia (1997). Sentencia SU-039/97, 47p.

Cortés, Raúl y Gómez, A. (2015). ¿De la especulación a la endogeneidad del crecimiento?: anotaciones críticas sobre economía aurífera y desarrollo regional en el departamento del Cauca. En: Investigación y Desarrollo, Vol. 23, No. 2, p. 278-311. doi: https://dx.doi. org/10.14482/indes.23.2.7169

Cortés, Raúl y Sinisterra, Mónica (2009). Colombia: capital social, movilización social y sostenibilidad del desarrollo en el Cauca. En: Revista CEPAL Vol. 99, p. 151-174.

Côte, Muriel y Korf, Benedict (2016). Making Concessions: Extractive Enclaves, Entangled Capitalism and Regulative Pluralism at the Gold Mining Frontier in Burkina Faso. En: World Development, Vol.101, January, p. 466-476. https://doi.org/10.1016/j.worlddev.2016.11.002

Duarte, Carlos; Solarte, Ana María; Rojas, Esneider; Salcedo, Leonardo y Rodríguez, Tania (2013). Análisis de la posesión territorial y situaciones de tensión interétnica e intercultural en el departamento del Cauca. Cali, Colombia: Convenio Universidad Javeriana Cali - Incoder, 163p.

Duarte, Bibiana; Urrea, Danilo; Cardona, Diego y Maldonado, Tatiana (2014). Extractivismo, conflictos y resistencias. Bogotá, Colombia: Censat Agua Viva - Amigos de la Tierra Colombia, 317p.

Falero, Alfredo (2015). La expansión de la economía de enclaves en América Latina y la ficción del desarrollo: siguiendo una vieja discusión en nuevos moldes. En: Revista Mexicana de Ciencias Agrícolas, p. 145-157.

Hanneman, Robert (1998). Computer-Assisted Theory Building: Modeling Dynamic Social Systems. California, Estados Unidos de América: Sage publications, 343p.

Harvey, David (2005). El nuevo imperialismo. Madrid, España: Ediciones Akal, 170 p. 
Hogenboom, Barbara; Baud, Michiel; de Castro, Fabio y Walter, Mariana (2014). La gobernanza ambiental en América Latina. Mapeando miradas, dinámicas y experiencias. En: Ecología política, No. 48, p 14-19.

Horning, Darwin; Bauer, Bernard y Cohen, Stewart (2016). Missing bridges: Social network (dis) connectivity in water governance. En: Redrafting Water Governance, No. 43, p. 59-70. https:// doi.org/10.1016/j.jup. 2016.06.006

Ille, Enrico (2016). Complications in the classification of conflict areas and conflicts actors for the identification of 'conflict gold' from Sudan. En: The Extractive Industries and Society, Vol. 3, No. 1, January, p. 193-203. doi: https://doi.org/10.1016/j.exis.2015.12.005

Jessop, Robert (2008). El futuro del estado capitalista. Madrid, España: Catarata, 432 p.

Mamián, Carlos Ariel; Velarde, Blanca Mercedes; Velasco, Deisy Lorena; Burbano, Sandra Ximena y Gómez, Ruth Beyra (2013). Prácticas de resistencia de las comunidades campesinas y organizaciones sociales en torno al agua, en contextos de La Vega y Sucre, macizo colombiano. Tesis para optar al título de magíster en Educación para la Diversidad. Universidad de Manizales, Popayán, Colombia, 76 p.

Martínez-Alier, Joan (2006). Los conflictos ecológico-distributivos y los indicadores de sustentabilidad. Polis. En: Polis, Revista de la Universidad Bolivariana Revista Latinoamericana, Vol. 5, No. 13, p. 3-12.

Merino, Roger (2015). The politics of extractive governance: Indigenous peoples and socio-environmental conflicts. En: The Extractive Industries and Society, Vol. 2, No. 1, p. 85-92. doi: https://doi.org/10.1016/j.exis.2014.11.007

Molano, Alfredo (2011). El Macizo Colombiano. Especial, El Espectador. Bogotá, Colombia.

Mondéjar, Remedios. (2015). Los conflictos ambientales y su tratamiento a través de la mediación. Madrid, España: Dykinson, 210 p.

Quiroga Martínez, Rayén (2007). Indicadores ambientales y de desarrollo sostenible: avances y perspectivas para América Latina y el Caribe. Santiago de Chile, Chile: Naciones Unidas, $228 \mathrm{p}$.

Ribeiro, Gustavo (2007). Poder, redes e ideología en el campo del desarrollo. En: Tabula Rasa, No. 6, p. 173-193.

Rodríguez, Julio César (2013). Cómo utilizar el Análisis de Redes Sociales para temas de historia. En: Signos Históricos, Vol. 15, No. 29, p. 102-141.

Scholz, John T; Berardo, Ramiro y Kile, Brad (2008). Do Networks Solve Collective Action Problems? Credibility, Search, and Collaboration. En: The Journal of Politics, Vol. 70, No. 2, p. 393-406. doi: $10.1017 / \mathrm{s} 0022381608080389$

Skorková, Zuzana (2016). Competency Models in Public Sector. En: Procedia - Social and Behavioral Sciences, Vol. 230, septiembre, 226-234. https://doi.org/10.1016/j.sbspro.2016. 09.029

Sovacool, Benjamin y Andrews, Nathan (2015). Does transparency matter? Evaluating the governance impacts of the Extractiv e Industries Transparency Initiative (EITI) in Azer- 
baijan and Liberia. En: Resources Policy, Vol. 45, p. 183-192. doi: https://doi.org/10.1016/j. resourpol.2015.04.003

Stiglitz, Joseph (2000). La economía del sector público. Barcelona, España: Antoni Bosch editor, $731 p$.

Stiglitz, Joseph (2010). Regulación y Fallas. En: Revista de Economía Institucional, Vol. 12, No. 1, p. 13-23.

Tocancipá-Falla, Jairo (2005). El retorno de lo campesino: una revisión sobre los esencialismos y heterogeneidades en la antropología. En: Revista Colombiana de Antropología, No. 41, p. 07-41.

Varela, Edgar (2014). El desarrollo regional como referente en el diseño e implementación de políticas públicas de promoción de paz: el caso del Macizo Colombiano y el alto Patía, en el sur del país. Bogotá, Colombia: Universidad de los Andes, Centro Interdisciplinario de Estudios sobre Desarrollo -Cider, 73p.

Walter, Mariana (2006). Conflictos ambientales, socioambientales, ecológico distributivos, de contenido ambiental. Reflexionando sobre enfoques y definiciones. En: Boletín ECOS Madrid, (febrero-abril), p. 5-25.

Walter, Mariana (2008). Nuevos conflictos ambientales mineros en Argentina. El caso Esquel (2002-2003). En: Revista Iberoamericana de Economía Ecológica, No. 8, p. 15-28.

Yagüe, Antonio José de Vicente (2010). Filosofía del "Buen Salvaje": Florello de Loaisel de Tréogate. En: Estudios Románicos, Vol. 19, p. 255-280.

Young, Michael; Peng, Mike; Ahlstrom, David; Bruton, Garry y Jiang, Yi (2008). Corporate Governance in Emerging Economies: A Review of the Principal-Principal Perspective. En: Journal of Management Studies, Vol. 45, No. 1, p. 196-220. https://doi.org/10.1111/j.14 $\overline{67-}$ 6486.2007.00752.x 


\section{ANEXO 1}

Universidad Del Cauca

Facultad de Ciencias Contables, Económicas y Administrativas

Departamento de Ciencias Económicas

Encuesta sobre Conflictividad, Redes Sociales y Gobernanza

(Nov. 03, 2015)

Municipio DE LA VEGA

- sta encuesta busca recopilar información sobre aspectos sociales y organizativos asociados a la

defensa del territorio y los recursos naturales. Su fin es estrictamente académico.

\section{ASPECTOS GENERALES}

1. ¿Conoce usted de actividades extractivas alrededor de recursos naturales?
a. Síl ）
b. No( )

2. ¿Cree usted que la realización de las actividades o proyectos mineros ha ocasionado problemáticas ambientales?
a. Sí ( )
b. No( )

3. Si es así, ¿cuál de las siguientes le parece más relevante?
a. Deforestación ( )
b. Contaminación hídrica ( )
c. Tratamiento de residuos ( )
d. Uso de suelos ( )
e. Otra ( ) ¿Cuál?

\section{REDES Y ACCIÓN COLECTIVA}

4. ¿A cuál organización pertenece usted?

Y, ¿dónde se encuentra ubicada?
a. LaVega ( )
b. LaSierra ( )
c. Otro ( ) ¿Cual?

5. ¿Cuál fue su año de fundación y objeto social?
a. Año
b. Objeto social

6. ¿Su organización ha sido consultada y/o convocada a discutir impactos de los proyectos minero auríferos en la región?
a. Sí ( )
b. No ( ) ¿Por quién?

7. ¿Su organización conoce de conflictos que involucran a los proyectos mineros de la zona?
a. Sí ( )
b. No( )
a.1 ¿Cuál? a. 2 ¿Dónde? 
8. ¿La organización a la que usted pertenece ha realizado acciones frente a la ejecución de estos proyectos?
a. Sí ( )
1. De ser así, ¿̇cuál?
2. ¿Dónde?
b. No( )

9. Si la respuesta a la pregunta anterior fue afirmativa, ¿iparticipa usted en actividades como proyectos o políticas que permitan una adecuada ejecución de las actividades extractivas?
a. Sí ( ) ¿Cuál(es)?
b. No( )

10. ¿Conoce usted grupos sociales, agencias u organismos que vigilen y monitoreen las actividades de las entidades encargadas de la ejecución de los proyectos?
a. Sí ( ) ¿Cuál(es)?
b. No( )

11. Si la respuesta a la pregunta anterior fue afirmativa, ¿̇a través de qué mecanismos?
a. Proyectos ( )
b. Acciones Legales ( )
c. Programas ( )

12. ¿Su organización ha participado activamente en la discusión de proyectos realizados en el municipio o la región en los últimos 10 años asociados a la minería del oro?
a. Sí ( ) ¿Cuál(es)?
b. No( )

13. ¿Con qué organizaciones o entidades se relacionó o asoció para enfrentar los problemas ambientales derivados de la actividad?

¿Cómo se vinculó?

14. ¿Considera que los objetivos propuestos fueron:
a) Totalmente alcanzados ( ) b. Parcialmente alcanzados ( )
c) No se consiguió ningún objetivo ( )

15. ¿Ha trabajado su organización con otras entidades (regionales, nacionales o globales), comunidades, grupos u organizaciones para gestionar proyectos o actividades en común?
a. Sí ( ) ¿Cuál(es)?
b. No( )

iMuchas gracias! 
ANEXO 2

\section{MEDIDAS DE COMPORTAMIENTO DE REDES SOCIALES}

\section{Densidad}

\begin{tabular}{lcccc}
\hline \multicolumn{4}{c}{ Density/Average matrix value } \\
\hline & 1 & 2 & 3 & 4 \\
& Density & No. of ties & Std de $v$ & avg de gree \\
\hline Red La Vega & 0,076 & 32 & 0,265 & 1,524 \\
\hline
\end{tabular}

Fuente: elaboración propia obtenida del procesamiento en el programa Ucinet.6 y la interfaz Netdraw 1.48

\section{Centralidad}

\begin{tabular}{llcc}
\hline & & Bonacich Eigenvector Centralities \\
\hline & & 1 & 2 \\
& & Eigenvec & $n$ Eigenvec \\
\hline 1 & Asoprocomqui & 0.000 & 0.000 \\
2 & Asoempreproagro & 0.000 & 0.000 \\
3 & Apimacizo & 0.162 & 22.963 \\
4 & Brisas del macizo & 0.000 & 0.000 \\
5 & Wairasitay & 0399 & 56.463 \\
6 & Vida mujer agri & $2.0 \mathrm{E}-0039$ & $2.8 \mathrm{E}-0037$ \\
7 & Asorposempmaci & 0.152 & 21.525 \\
8 & Asap & 0.404 & 57.135 \\
9 & Abriendo trocha & 0.399 & 56.463 \\
10 & Conciencia campesina & 0.382 & 53.971 \\
11 & Proceso campesino & 0.348 & 49.275 \\
12 & Pucsoc & 0.250 & 35.421 \\
13 & Cima & 0.319 & 45.061 \\
14 & Alcaldía & 0.000 & 0.000 \\
15 & Gobernación & 0.030 & 4.214 \\
16 & Crc & 0.104 & 14.699 \\
17 & Cabildos indígenas & 0.073 & 10.361 \\
18 & Identidad campesina & 0.073 & 10.361 \\
19 & Astanta & 0.070 & 9.904 \\
20 & Asocampesinatraba & 0.070 & 9.904 \\
21 & Asocampesinapatia & 0.070 & 9.904 \\
\hline & & & \\
\hline
\end{tabular}

\begin{tabular}{llcc}
\hline & & \multicolumn{2}{c}{ Descriptive Statistics } \\
\hline & & $\begin{array}{c}1 \\
\text { Eigenvec }\end{array}$ & $\begin{array}{c}2 \\
\text { Eigenvec }\end{array}$ \\
\hline 1 & Mean & 0.157 & 22.268 \\
2 & Std Dev & 0.151 & 21.366 \\
3 & Sum & 3.307 & 467.624 \\
4 & Variance & 0.023 & 456.525 \\
5 & SSQ & 1.000 & 20000.002 \\
6 & MCSSO & 0.479 & 9587.030 \\
7 & Euc Norm & 1.000 & 141.421 \\
8 & Minimum & 0.000 & 0.000 \\
9 & Maximum & 0.404 & 57.135 \\
10 & N of Obs & 21.000 & 21.000 \\
11 & N Missing & 0.000 & 0.000
\end{tabular}

Network centralization index - 47.15\%

Fuente: elaboración propia obtenida del procesamiento en el programa Ucinet.6 y la interfaz Netdraw 1.48 
Cercanía

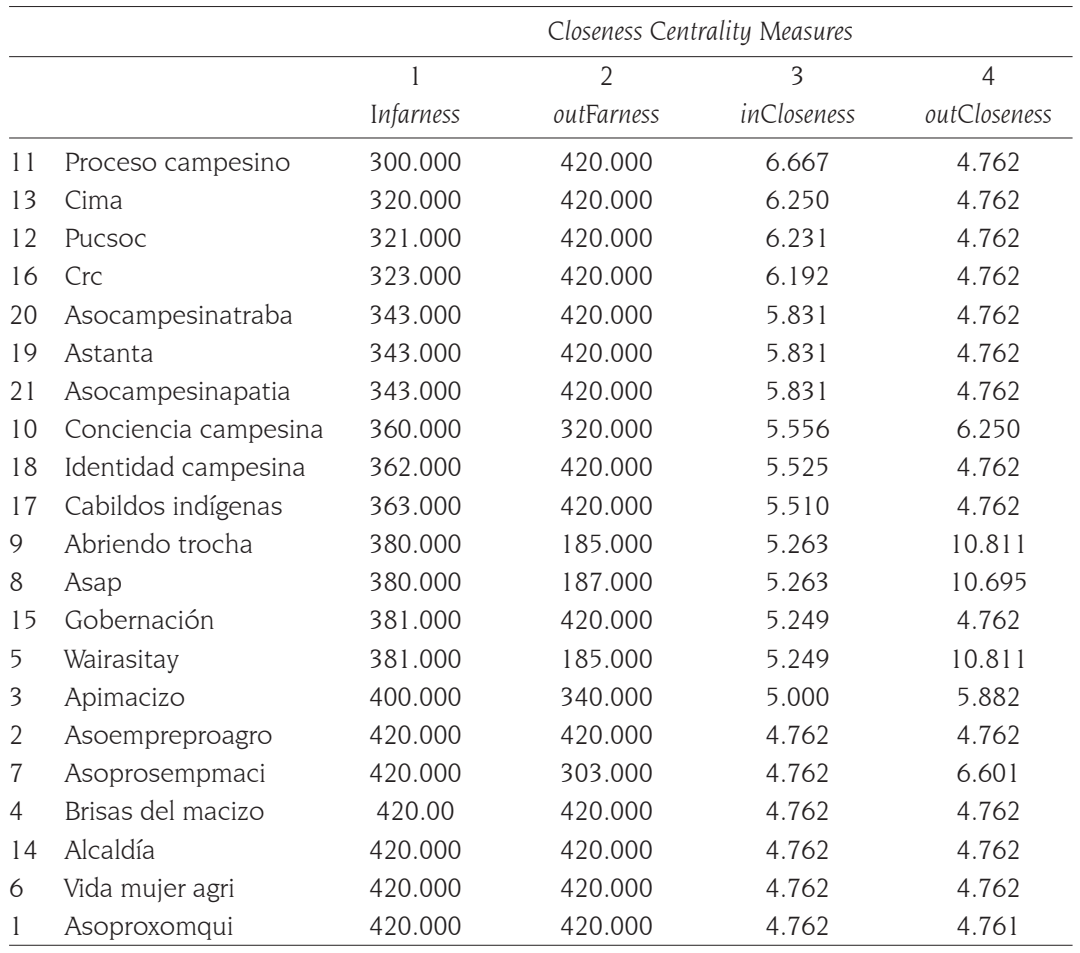

\begin{tabular}{|c|c|c|c|c|c|}
\hline & & \multicolumn{4}{|c|}{ Statistics } \\
\hline & & 1 & 2 & 3 & 4 \\
\hline & & Infarness & outFarness & inCloseness & outCloseness \\
\hline 1 & Minimum & 300 & 185 & 4,762 & 4,762 \\
\hline 2 & Average & 372,381 & 372,381 & 5,429 & 5,832 \\
\hline 3 & Maximum & 420 & 420 & 6,667 & 10,811 \\
\hline 4 & Sum & 7820 & 7820 & 114,018 & 122,478 \\
\hline 5 & Standard Deviation & 38,193 & 83,725 & 0,574 & 2,084 \\
\hline 6 & Variance & 1458,712 & 7009,950 & 0,330 & 4,342 \\
\hline 7 & SSQ & 2942652 & 3059228 & 625,977 & 805,504 \\
\hline 8 & MCSSQ & 30632,953 & 147208,953 & 6,920 & 91,172 \\
\hline 9 & Euclidean Norm & 1715,416 & 1749,065 & 25,020 & 28,381 \\
\hline 10 & Observations & 21 & 21 & 21 & 21 \\
\hline 11 & Missing & 0 & 0 & 0 & 0 \\
\hline
\end{tabular}

Fuente: elaboración propia obtenida del procesamiento en el programa Ucinet.6 y la interfaz Netdraw 1.48 


\section{Intermediación}

\begin{tabular}{|c|c|c|c|}
\hline \multicolumn{4}{|c|}{ Freeman Betweenness Centrality } \\
\hline & & 1 & 2 \\
\hline & & Betweenness & Betweenness \\
\hline 10 & Conciencia campesina & 9.000 & 2.368 \\
\hline 9 & Abriendo trocha & 4.000 & 1.053 \\
\hline 3 & Apimacizo & 3.000 & 0.789 \\
\hline 8 & Asap & 2.000 & 0.526 \\
\hline 5 & Wairasitay & 2.000 & 0.526 \\
\hline 1 & Asoprocomqui & 0.000 & 0.000 \\
\hline 7 & Asoprosempmaci & 0.000 & 0.000 \\
\hline 2 & Asoempreproagro & 0.000 & 0.000 \\
\hline 4 & Brisas del macizo & 0.000 & 0.000 \\
\hline 6 & Vida mujer agri & 0.000 & 0.000 \\
\hline 11 & Proceso campesino & 0.000 & 0.000 \\
\hline 12 & Pucsoc & 0.000 & 0.000 \\
\hline 13 & Cima & 0.000 & 0.000 \\
\hline 14 & Alcaldía & 0.000 & 0.000 \\
\hline 16 & Gobernación & 0.000 & 0.000 \\
\hline 17 & Cabildos indígenas & 0.000 & 0.000 \\
\hline 18 & Identidad campesina & 0.000 & 0.000 \\
\hline 19 & Astanta & 0.000 & 0.000 \\
\hline 20 & Asocampesinatraba & 0.000 & 0.000 \\
\hline 21 & Asocampesinapatia & 0.000 & 0.000 \\
\hline
\end{tabular}

\begin{tabular}{|c|c|c|c|}
\hline \multicolumn{4}{|c|}{ Descriptive Statistics for each measure } \\
\hline & & 1 & 2 \\
\hline & & Betweenness & Betweenness \\
\hline 1 & Mean & 0.952 & 0.251 \\
\hline 2 & Std Dev & 2.126 & 0.560 \\
\hline 3 & Sum & 20.000 & 5.263 \\
\hline 4 & Variance & 4.522 & 0.313 \\
\hline 5 & SSO & 114.000 & 7.895 \\
\hline 6 & MCSSQ & 94.952 & 6.576 \\
\hline 7 & Euc Norm & 10.677 & 2.810 \\
\hline 8 & Minimum & 0.000 & 0.000 \\
\hline 9 & Maximum & 9.000 & 2.368 \\
\hline
\end{tabular}

Fuente: elaboración propia obtenida del procesamiento en el programa Ucinet.6 y la interfaz Netdraw 1.48 